\title{
Loss of Calculation in Nature Disaster by using Laplacian of Gaussian
}

\author{
Sushma Yadav \\ Sushmayadav1249@gmail.com \\ M.Tech (IT), Gitam University \\ Visakhapatnam, India
}

\begin{abstract}
In last few years, the nature Disaster was occur in many counties that cause on building, Hospital, university etc. in such that cases it can't calculate the loss of economy. For that it will take a survey on that disaster place, so then it can calculate the loss of economy. In this paper, the loss of calculation in nature disaster is calculated by using Laplacian of Gaussian algorithm.
\end{abstract}

\section{Keywords}

Sobel operator, 3D edge detector

\section{INTRODUCTION:}

From last few years, natural disaster occurs in various countries which hamper a lot of geographical and building losses. A natural disaster is a major adverse event resulting from natural processes of the Earth; examples include floods, volcanic eruptions, earthquakes, tsunamis, and other geologic processes. A natural disaster can cause loss of life or property damage, and typically leaves some economic damage in its wake. Then there is loss of property, which affects people's living quarters, transportation, livelihood, and means to live. Fields saturated in salt water after tsunamis take years to grow crops again. Homes destroyed by floods, hurricanes, cyclones, landslides and avalanches, a volcanic eruption, or an earthquake are often beyond repair or take a lot of time to become liveable again. Personal effects, Memorabilia, vehicles, and documents also take a hit after many natural disasters. In 2012, there were 905 natural disasters worldwide, $93 \%$ of which were weather-related disasters. Overall costs were US $\$ 170$ billion and insured losses $\$ 70$ billion. 2012 was a moderate year. $45 \%$ were meteorological (storms), 36\% were hydrological (floods), $12 \%$ were climatological (heat waves, cold waves, droughts, wildfires) and 7\% were geophysical events (earthquakes and volcanic eruptions). Between 1980 and 2011 geophysical events accounted for $14 \%$ of all natural catastrophes. The natural disasters that really affect people worldwide tend to become more intense as the years go on. Frequency of earthquakes, mega storms, and heat waves has gone up considerably in the last few decades. Heavy population

In areas that get hit by floods, cyclones, and hurricanes has meant that more lives are lost. In some areas, the population has gotten somewhat prepared for the eventuality of disasters and shelters are built for hurricanes and tornadoes. However, loss of property is still a problem, and predicting many natural disasters isn't easy. Scientists, geologists, and storm watchers work hard to predict major disasters and avert as much damage as possible. With all the technology available, it's become easier to predict major storms, blizzards, cyclones, and other weather related natural disasters. But there are still natural disasters that come up rather unexpectedly, such as earthquakes, wildfires, landslides, or even volcanic eruptions. Sometimes, a time of warning is there, but it's often very short with catastrophic results. Areas that are not used to disasters affected by flash floods or sudden hail storms can be affected in an extreme way.

\section{RELATED WORK}

\subsection{Image noise reduction: \\ Median Filter}

The median filter is normally used to reduce noise in an image and it is a simple and very effective noise removal filtering process. Noise is removed by replaces the center value with the median of all the pixel values in the matrix. If we considering an example of $3 \times 3$ matrix.

Table 1

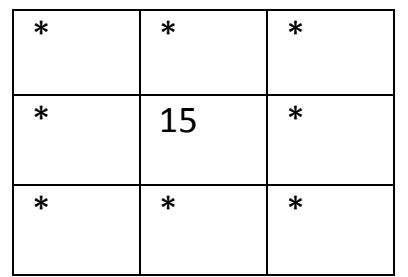

Table 2

\begin{tabular}{|l|l|l|}
\hline 11 & 15 & 19 \\
\hline 12 & 17 & 18 \\
\hline 14 & 16 & 13 \\
\hline
\end{tabular}

The median filter sorts the value of the given matrix and then median value is replace by center value. The sorted values are $11,12,13,14,15,16,17,18,19$ and median value 15 will be replaced by centre value in the matrix. This process is performed over the whole image and reduces the noise.

\subsection{Sobel operator: [1]}

The Sobel operator, sometimes called Sobel Filter, is used in image processing and computer vision, particularly within edge detection algorithms, and creates an image which emphasizes edges and transitions. In this paper the sobel operator is used for detection of ships names. The Sobel operator is based on convolving the image with a small, separable, and integer valued filter in horizontal and vertical direction and is therefore relatively inexpensive in terms of computations. The operator uses two $3 \times 3$ kernels which are convolved with the original image to calculate approximations of the derivatives - one for horizontal changes, and one for vertical. If we define $\mathbf{A}$ as the source image, and $\mathbf{G}_{x}$ and $\mathbf{G}_{y}$ are two images which at each point 
contain the horizontal and vertical derivative approximations, the computations are as follows

$$
\Delta f=\operatorname{mag}(\Delta f)=\left[G_{x}^{2}+G_{y}^{2}\right]^{\wedge} 1 / 2
$$

\subsection{A 3D edge detection scheme [2] Filtering stage}

Choice of 1D smoothing operator: $T(X)$

We strongly recommend to choice a filter that can be implemented recursively, mainly because of the computing time. We can for example choose one of two filters: $T_{1}$ or $T_{2}$.

$$
\begin{aligned}
& T_{1}(\mathrm{x})=\mathrm{ce}^{-\alpha|\mathrm{x}|} \\
& T_{2}(\mathrm{x})=(\mathrm{c}|\mathrm{x}|+1) \mathrm{e}^{-\alpha|\mathrm{x}|}
\end{aligned}
$$

Theoretically the derivation filter $T_{2}^{\prime}(\mathrm{x})$ is better than Canny's multiple response criterion, but $T 1(x)$ meets the best trade-off detection location. For small value $T_{2}^{\prime}(\mathrm{x})$ induce some delocalization problem. This drawback will be share to any filter whose impulse response is at point 0 . Or else the results do not different on many kinds of image.

\subsection{Choice of the Kind of Approach}

Generally second derivative of computation is more sensible to noise. The simplification is occure that's why the computational cost is low, when computing the impulse response of the filter by multiplication and addition of smoothing and second derivative operator. Generally image

noisy is used for threshold zero crossing provided by the filtering stage and this require to gradient magnitude to zero crossing. There are two kind of method for edge localization is that experimentally the same, it may be

point out that laplacian approach tends to smooth the right angle.

Let $\mathrm{I}(\mathrm{X} 1, \ldots \ldots . \mathrm{Xn})$ image dimension $\mathrm{n}$.

Let $\mathrm{G}(\mathrm{X} 1, \ldots \ldots . \mathrm{Xn})$ Gradient of I.

$$
G(I)=\left(\frac{\partial I}{\partial X 1}, \ldots \ldots \frac{\partial I}{\partial X n}\right)^{t}
$$

The computation of the gradient component $\frac{\partial I}{\partial X 1}$ is done by computing image $\left(D_{i}\right)$ corresponding to the partial derivatives with respect to $X_{i}$ as follow

$$
\begin{aligned}
& \text { For } \mathrm{i}=1, \ldots \mathrm{n} \text { do } \\
& D_{i}=\mathrm{I} \\
& \text { For } \mathrm{j} \text { e }[1, \ldots . \mathrm{n}] \backslash\{\mathrm{i}\} \text { do } \\
& D_{i}=D_{i} * \mathrm{~T}\left(X_{j}\right) \\
& D_{i}=D_{i} * T^{\prime}\left(X_{1}\right)
\end{aligned}
$$

For an image of size $\mathrm{p}$ (ie $d_{1} * \ldots * d_{p}$ ) the computation of each gradient component needs to compute $p$ convolutions per point. The we obtain for the entire computation $p^{2} . \Pi_{x=1}^{p} d_{i}$. convolutions. If we use a direct implementation of a convolution mask ID of size k, we obtain the following complexity: $k p^{2} \Pi_{x=1}^{p} d_{i}$. A recursive filtering of order $r$ allows to obtain a complexity of order: $r p^{2} \prod_{x=1}^{p} d_{i}$. The computation of the second derivative may be done with the algorithmic structure precedently described where the first derivative operator $\mathrm{T}^{\prime}(\mathrm{x})$ is replaced by the second derivative operator $T^{\prime \prime}(x)$. However for some cases calculus simplification occur allowing to reduce the computational cost

\section{PROPOSED WORK:}

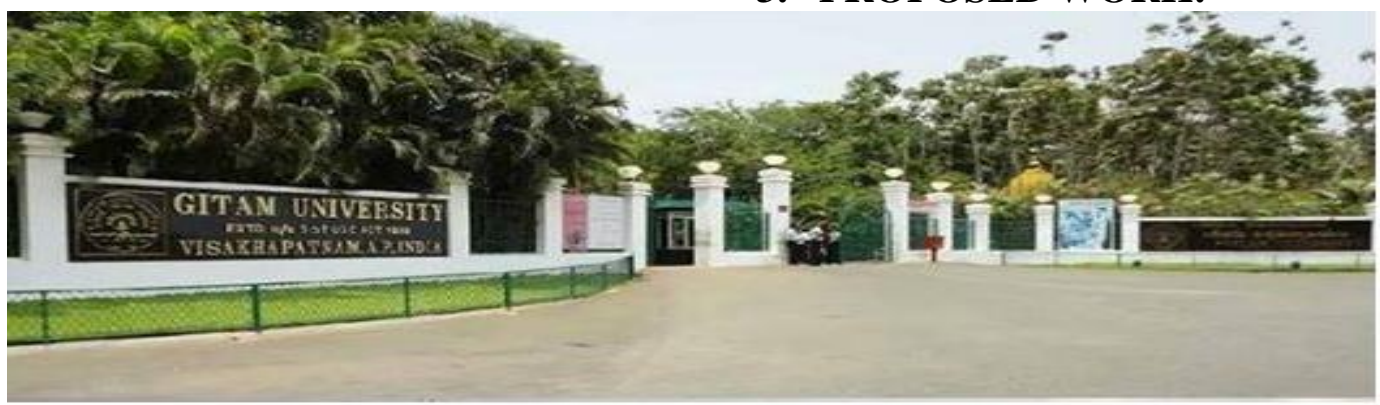

Figure 1. Before Disaster

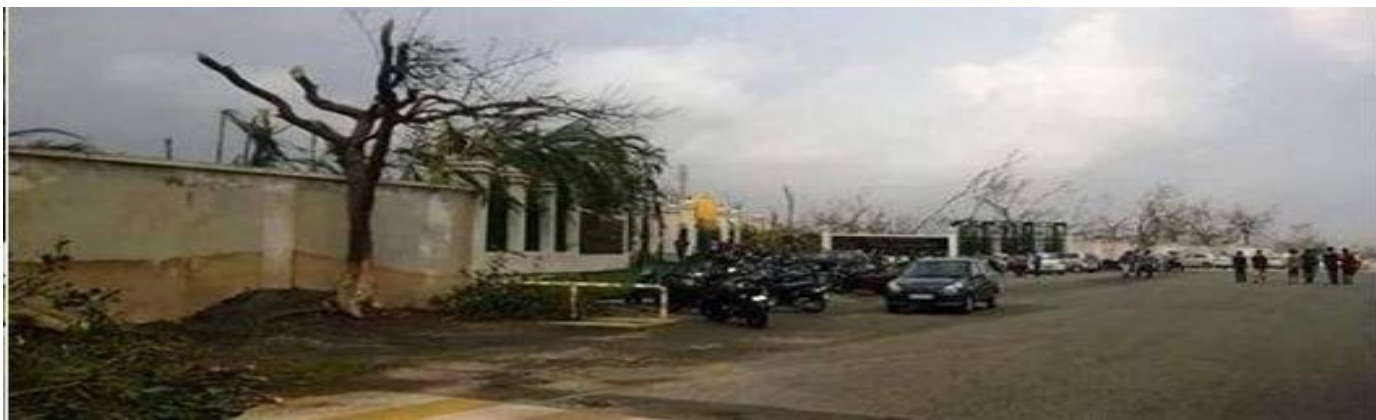

Figure 2. After Disaste 


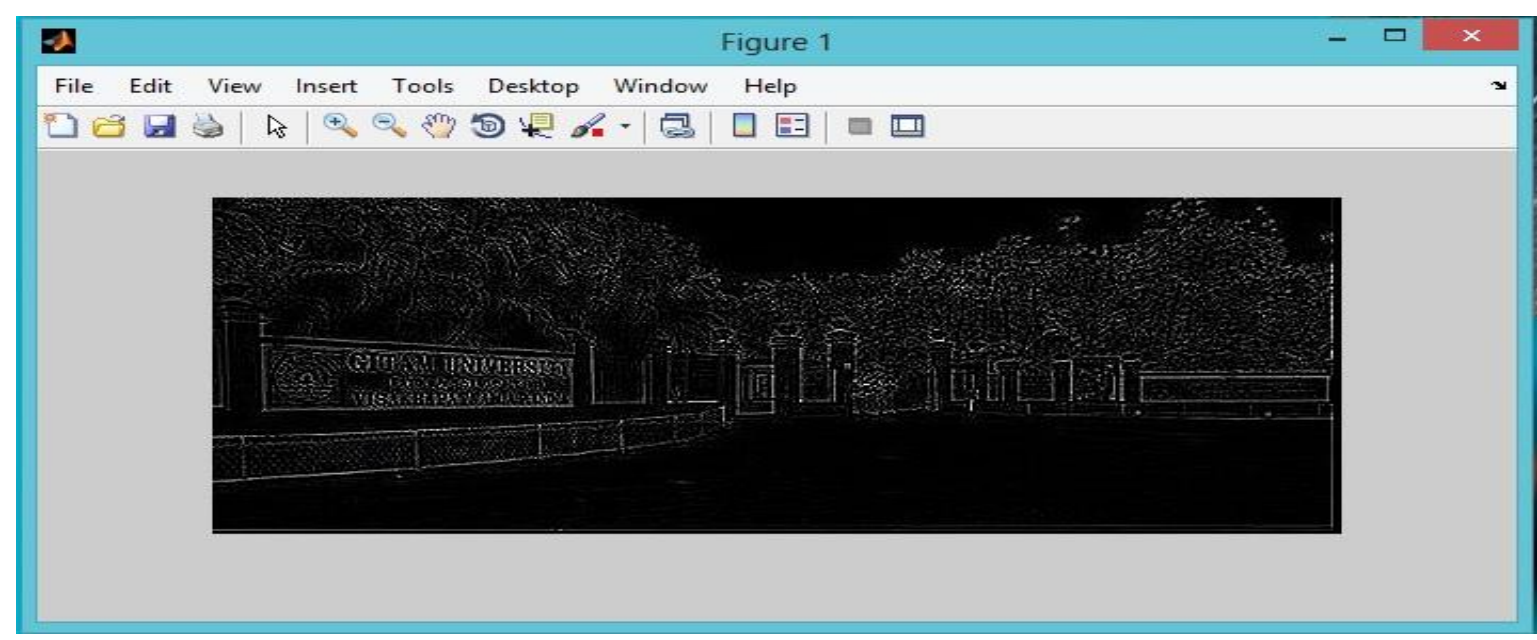

Figure 3. Output of Before Disaster

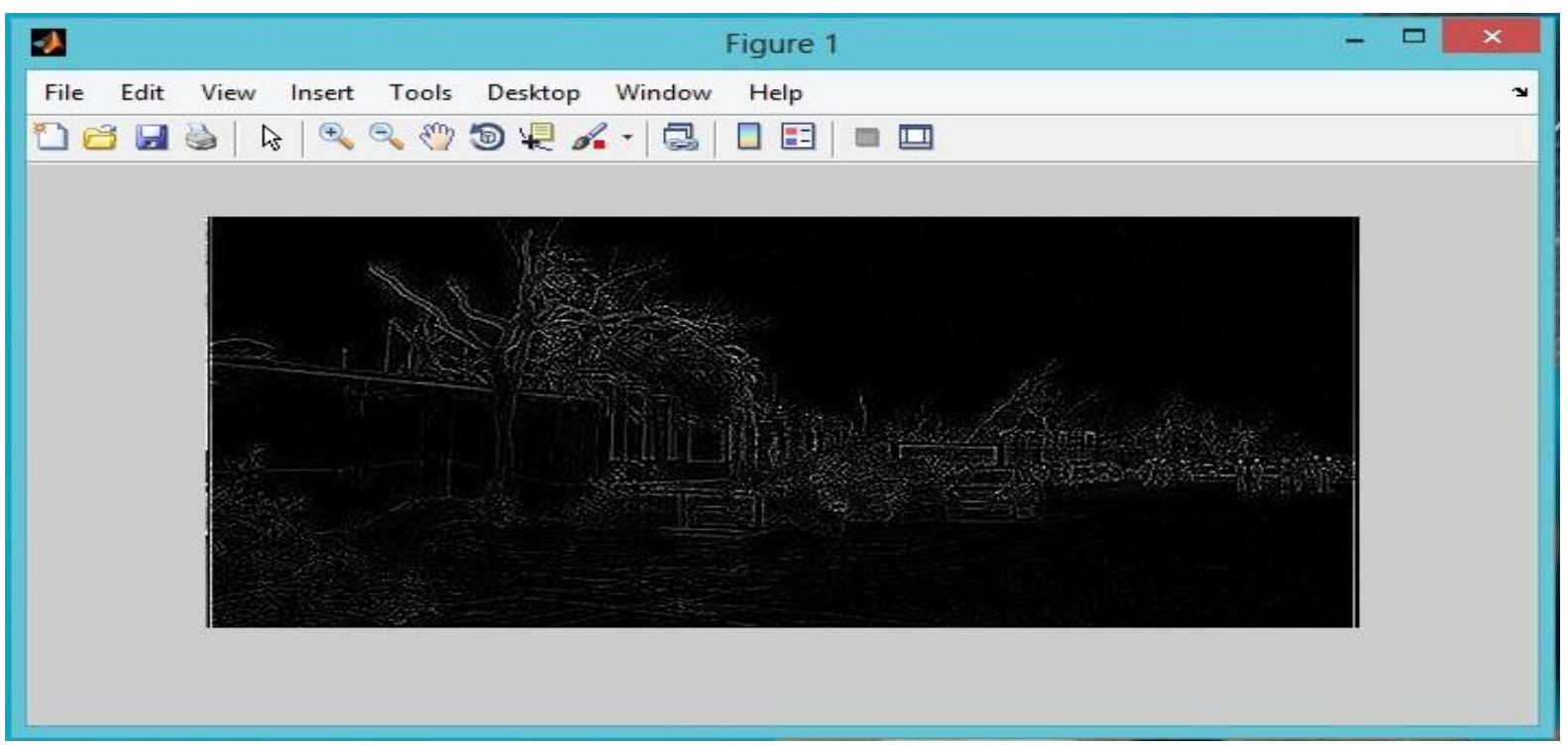

Figure 4. Output of After Disaster

\section{Step 1:}

In this paper, the loss of nature disaster calculated by using Laplacian of Gaussian algorithm. The first stage is that the camera will take a photo (2D or 3D) then if in that image if it is noise image then image noise reduction process will start.

\section{Step 2:}

The Sobel operator is mainly used for detection of name. The Sobel operator are mainly based on convolving the image with a small, separable and integer value filter in horizontal and vertical direction. In this paper, camera will take a $2 \mathrm{D}$ or $3 \mathrm{D}$ photo. When camera will take $3 \mathrm{D}$ photo then it will filter the 1D smoothing operators.

\section{Step 3:}

At final stage, Before Disaster figure 3 and After Disaster figure 4 will compare by using Laplacian of Gaussian algorithm. And it will calculate the loss of economy in nature disaster.

\section{ACKNOWLEDGEMENTS}

The author would like to thank K. N. Soujanya from Gitam University for various help

\section{REFERENCES}

[1] "Stealth Ships Detection by using Laplacian of Gaussian Algorithm", Sagar Shankarrao Dake

[2] M.Tech (IT), Gitam University Visakhapatnam, India

[3] "3D edge detection by separable recursive filtering and edge closing" Olivier MONGAINRIA ,Doinaine de Voluceau - B.P. 105, Rachid DERICHE INRIA Sophia-Antipolis - 2004, Route des Lucioles06565 VALBONNE CEDEX - FRANCE ,Gregoire MALANDAIN INRIA - Doniaine de Voluceau - B.P. 10578153 LE CHESNAY CEDEX - FRANCE ,Jean Pierre COCQUEREZ ENSEA - Inipasse des ch2nes pourpres 95014 CERGY PONTOISE FRANCE 78153 LE CHESNAY CEDEX FRANCE. 